\title{
A Fast Lane Detection and Tracking Algorithm
}

\author{
DONG Hui-fen ${ }^{1,}$ a , HU Ming-bo ${ }^{2, b}$ \\ ${ }^{1}$ Civil Aviation University of China, Dongli Distric, Tianjin, China \\ ${ }^{2}$ Civil Aviation University of China, Dongli Distric, Tianjin, China \\ a758723287@qq.com, b254748523@qq.com
}

Keywords: lane; linear model; coordination detection; Hough transformation; directional filter Abstract: To improve the real time performance and robustness, an algorithm about lane coordination detection is proposed. The lane parameter is detected by the local windows extraction and the global extraction based on Hough transformation, with the help of the lane parameter confidence interval and the active box of interest. The algorithm is conducted including the below steps. First, set two groups of active boxes of interest; second, filter directionally; third, scan the number of lines in the interest box. The candidate points are selected to fit a straight line if the points have the max gradient. The experimental results show that the proposed scheme is accurate, robust and fast enough for the requirements of real time.

\section{Introduction}

In the lane migration system, the precision and speed of the lane line detection are the most important. In recent years, for the lane line visual detection problem, researchers have proposed many different algorithms, such as the method based on feature and the method based on model. Through the analysis of image low-level features such as edge, the method based on feature can split out the road from the image, and recognize the lane line ${ }^{[1-2]}$. Lane line can be described by a small number of model parameters, if the shape of the lane line is a straight line or parabola and calculation process of the model parameters can be seen as the process of lane detection ${ }^{[3-4]}$. Due to the interference of the vehicle, shadow, water and other factors, the method based on the characteristics is only applicable to such a situation that the road surface is clean and the light is good. At the same time, this method can not eliminate the interference of vehicles or noise. Although the model-based method has stronger robustness than that based on feature, but because of onerous computation and complex parameter optimization, the method is difficult to meet the real-time requirements of the intelligent vehicle navigation.

Under the low speed environment, the camera preview distance doesn't need to be too great. Within a certain foresight, the road can be considered to be linear, so the linear model is adopted. Coordinating the global parameters extraction of lane line based on Hough transform and the local parameter extraction of lane line based on least square fitting, the time detecting lane line is reduced.

\section{The overall algorithm of the lane line detection}

First of all, it needs to determine whether the collected image is the first frame. If it is the first frame image, the image is preprocessed. The left and right lane lines are detected by using the lane line extraction algorithm based on Hough transform. Otherwise, the left and right edges are processed by using two groups of interested active box to extract candidate and to fit a straight line. Then, the calculated lane line slope of the current frame is compared with that of the last frame. If 
the results do not meet the requirements of the confidence interval, the system will put the next frame image as the first frame to deal with and reuse lane line extraction algorithm based on Hough transform to detect the lane line. The above process is shown as Fig. 1.

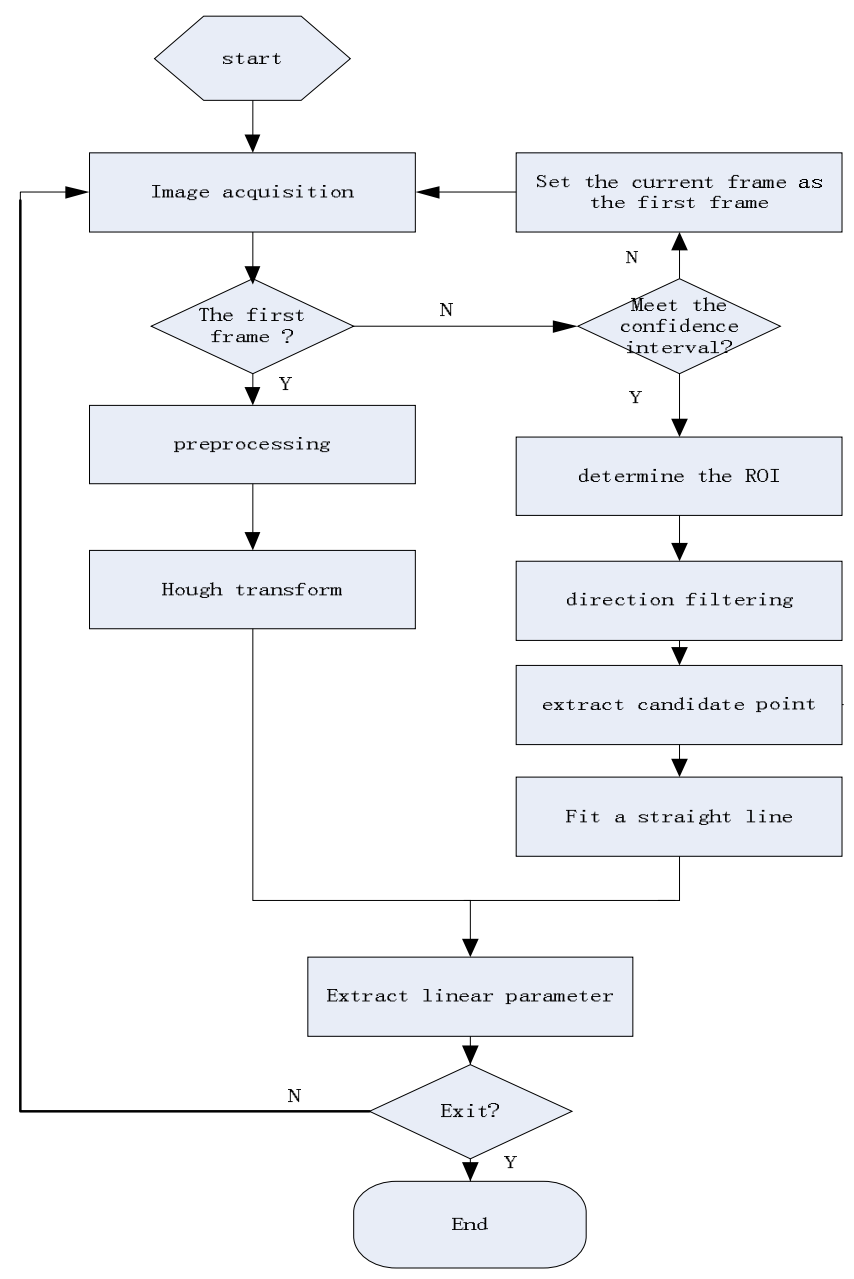

Fig. 1 Overall algorithm flow chart

\section{Lane line detection}

\section{Image preprocessing}

First, the collected image is processed by graying and median filtering. Then the edge image is obtained by canny edge detection. There is often a lot of useless edge information on both sides of the road. If all the edge information is dealt directly with, it can cause unnecessary operations and bring more difficulties to road detection. Cutting edge noise can filter useless edge points. The effect of the edge noise suppression is shown in Fig. 2.

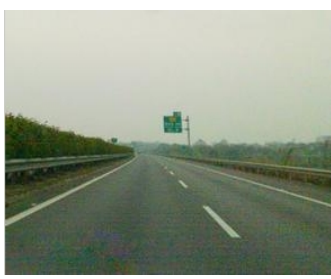

(1) the input image

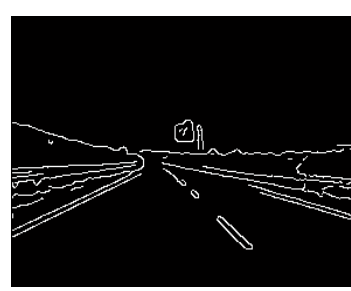

(2) edge graph

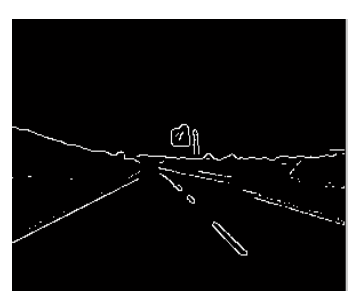

(3) the edge inhibition of figure

Fig. 2 the effect of the edge noise suppression 


\section{The extraction of straight line}

Hough transform is as a result of the straight line segments, and the non-road line segments are more than the road line segments. Lane boundary can be selected from a line segment set by using a set of constraint condition.

\section{Relative angle constraint}

The actual situation of traffic can get the following assumptions:

1> Car is in the driveway;

2> Angle that the car is relative to the lane line is in a reasonable range;

Based on the above assumptions, line segments describing the lane boundary are limited in the scope of the general location and slope in the image coordinate system. If the slope of straight line is between $\mathrm{k}_{\min }$ and $\mathrm{k}_{\max }$, it may be the lane line; otherwise the line segment is interference.

\section{Interframe continuity constraint}

The slope and intercept can determine a straight line. The interframe continuity of lane boundary requires that the change of the slope $\mathrm{k}$ and intercept $\mathrm{b}$ between two adjacent frames is in a reasonable scope, which is shown in equation (1):

$$
\left\{\begin{array}{l}
\left|b_{i}-b_{p r e}\right|<\Delta b_{\max } \\
\left|\arctan \left(k_{i}\right)-\arctan \left(k_{p r e}\right)\right|<\Delta \phi_{\max }
\end{array}\right.
$$

In the equation, $k_{i}$ and $b_{i}$ are the slope and intercept of the $i$-th segment. $k_{\text {pre }}$ and $b_{\text {pre }}$ are the slope and intercept of the lane boundary. $\Delta \psi_{\max }$ and $\Delta \mathrm{b}_{\max }$ are the maximum tolerance variation of the slope and intercept.

\section{Area and the length of the constraints}

Area constraint, that is, when there are multiple line accords with a condition, choose the line closest to the central image as the lane line. Because the interference usually come from the outside area of road, choosing the line closest to the central image as a lane boundary is reasonable. Length constraint, namely length must meet the minimum value setted.

\section{Lane line tracking}

\section{Interest area}

In this paper, Interest area is selected by two groups of interested active boxes (ABOI) ${ }^{[8]}$, which is shown in Fig. 3. Track road right edge ABOI by the following set of equations for iteration.

$$
\left\{\begin{array}{l}
x_{j}^{R}=x_{j-1}^{R}-h_{j-1}^{R}(1+\lambda)\left(2 k^{R}\right)-b_{j-1}^{R}(1-\lambda) / 2 \\
b_{j}^{R}=\lambda b_{j-1}^{R} \\
h_{j}^{R}=\lambda h_{j}^{R} \\
y_{j}^{R}=y_{j-1}^{R}-h_{j}^{R}
\end{array}\right.
$$

In the equation, $x_{j}{ }^{R}$ and $y_{j}{ }^{R}$ are the abscissa and ordinate of the left vertex of the $j$-th box of interest. $h_{j}{ }^{R}$ and $b_{j}{ }^{R}$ are the height and width of the left vertex of the $j$-th box of interest. $x_{0}{ }^{R}$ and $\mathrm{y}_{0}{ }^{\mathrm{R}}$ are the intersection point that belong to the right boundary and image boundary in the last frame. $\lambda$ is the attenuation factor, generally between $0.6 \sim 0.95 . \mathrm{k}^{\mathrm{R}}$ is the slope of the border on the right side of the road. The calculation method of left AOBI group is similar to the right. 


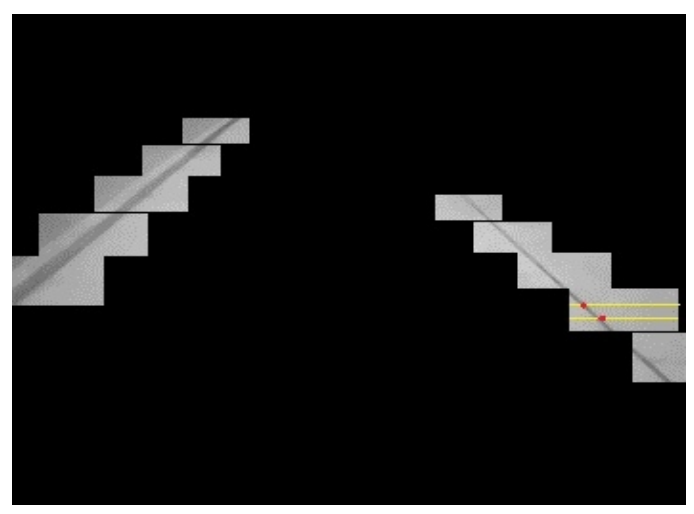

Fig. 3 Scannogram based on interest box

The selection of candidate points

Grayscale of road is usually evenly. The Lane line has high grey value relative to the road, as shown in Fig. 4:

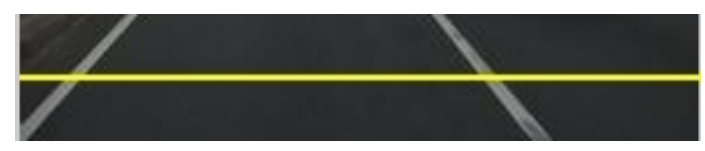

(1) the input image

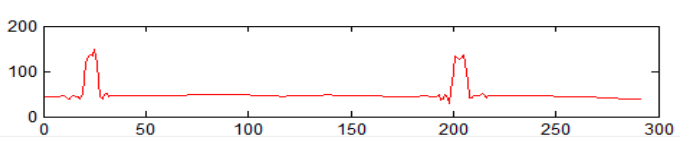

(2) the grayscale

Fig. 4 the grayscale of yellow line

\section{The orientation filter}

Because the relative to the road the lane line has high grey value, it can be detected by the method of threshold value. But when the light changes, the effect of this method is not ideal. So the lane line can be detected by the sensitivity to gray scale difference value. Direction gradient operator can satisfy this requirement. The effect of the direction gradient operator in $0{ }^{\circ} 、 45^{\circ}$ 、 $180^{\circ} 、 135^{\circ}$ is shown in Fig 5. It can be seen that $0^{\circ}$ and $180^{\circ}$ direction gradient operator in the $45^{\circ}$ and $135^{\circ}$ direction have very good effect; two kinds of the direction gradient operators can be used.

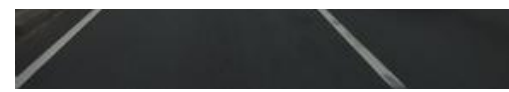

(a)The input image

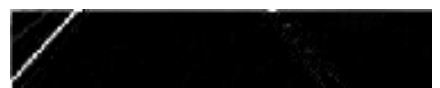

(b) $0^{\circ}$

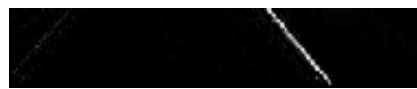

(d) $135^{\circ}$

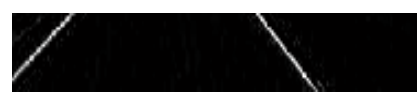

(c) $45^{\circ}$

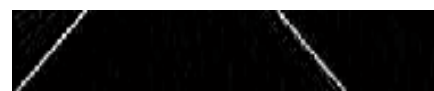

(e) $180^{\circ}$

\section{Search in interested active boxes}

Fig. 5 direction filtering figure in $0^{\circ} 、 45^{\circ} 、 180^{\circ} 、 135^{\circ}$

After computing the gradient operator with the graphics, one-third and two-thirds of the line of interested active boxes is scanned, this is shown in Fig 3. A maximum gradient of points for a candidate in the scan line is selected. When the lane line is interval, some interest box may have not lane line, the extracted points are not needed. The gradient value of the point on lane line is more often than the gradient value of the point on non-lane line. A threshold value is settled, if the candidate gradient value is greater than the threshold, the point is extracted. Otherwise it is abandoned. The overall search steps are as follows:

Step1: Scan the line of the right interested active boxes, extract the 10 candidate points; 
Step2: Compare grey value of 10 candidate points with threshold value, the point which grey value is greater than the threshold is filtered;

Step3: If the selected points are not less than two points, these points are linear fitted linearly. If the selected points are less than two, the current frame is settled as the first frame, and the next time process the whole image;

Step4: The process of the left side is same as the right;

\section{The confidence interval}

The slope changes of two consecutive frames are generally in a certain range. If the slope changes of two consecutive are out of range, it can be considered that the detected line does not conform to the requirements. So the slope of the straight line $\mathrm{k}_{1}$ of the current frame is compared with the slope of the straight line $\mathrm{k}_{2}$ of the last frame. If $\left|\mathrm{k}_{1-} \mathrm{k}_{2}\right|<\Delta \mathrm{k}$, the extracted the lane line is practical lane line. Otherwise, the current frame is as the first frame and process the overall image.

\section{The experimental results and analysis}

The algorithm in this article is based on VC environment and OpenCV image processing library. Experiment is conducted on a road in the university, and 1000 lane frames in a video image sequence is intercepted and analyzed. After image processing, there are 976 images accurately detecting the lane line, the detection accuracy is about $97.6 \%$. As a result of too big jump of lane line of the two consecutive frames or too serious interference information, the detection error appears.

Real-time is also one of the important indices for measuring algorithm performance. The image size processed in this paper is $352 \times 288$. It takes about $50 \mathrm{~ms}$ to operate the algorithm in the lane line detection link, and takes about $24 \mathrm{~ms}$ in the lane line tracking link. On average the time of every frame image processing is between $24 \sim 37 \mathrm{~ms}$, so the algorithm can meet the demands of practical application.

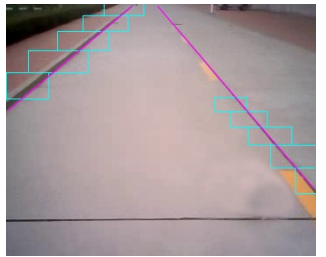

(1) 81 frames

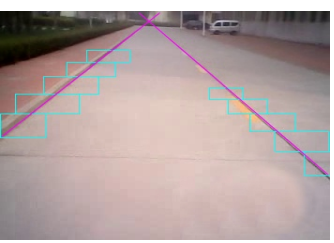

(3) 487 frames

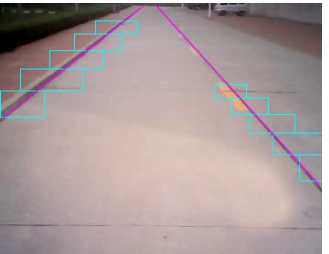

(2) 276 frames

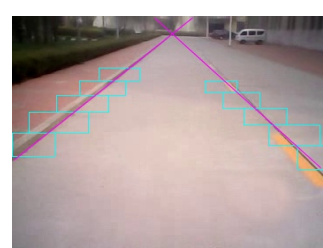

(4) 789 frames

Fig. 64 lane line extraction figure

\section{Conclusion}

This paper proposes a real-time method based on linear model to detect lane line. Due to the parameter confidence interval, active box of interest, and the coordination of global parameters extraction of lane line based on Hough transform and local parameter extraction of lane line based on linear fitting, real-time and robustness of the lane line detection is improved. Because edge 
detection and Hough transform are effectively avoided in the tracking link of lane line, the running speed of algorithm is greatly improved. The experimental results show that for different road conditions, the algorithm proposed in this paper has better real-time and robustness.

\section{References:}

[1] Wang J G, Lin C J, Chen S M. Applying fuzzy method to vision-based lane detection and departure warning system [J]. Expert Systems with Applications(S0957-4174), 2010, 37(1): 113-126.

[2]Gao Qingji, Luo Qijun, Sun Moli. Rough set based unstructured road detection through feature learning $[\mathrm{J}]$. Proceedings of the IEEE International Conference on Automation and Logistics, Jinan, China. 2007(8): 101-106

[3] Lopez A, Serrat J, Canero C, et al. Robust lane markings detection and road geometry computation [J]. International Journal of Automotive Technology(S1229-9138), 2010, 11(3): 395-407.

[4]Franke U, Loose H, Knöppel C. Lane recognition on country roads[J]. Proceedings of the 2007 IEEE Intelligent Vehicles Symposiumb Istanbul， Turkey. 2007(7): 99-104

[5]Ramstrom $\mathrm{O}$ and Christensen $\mathrm{H}$. A method for following unmarked roads[J]. IEEE. 2005: 650-655

[6]Wang Jian, Ji Zhong. Unstructured road detection using hybrid features[J]. IEEE Proceedings of the Eighth International Conference on Machine Learning and Cybernetics, Baoding. 2009: 482-486 [7] Saudi Azali, Teo Jason. Hierarchical additive Hough transform for lane detection[J]. IEEE Embedded Systems Letters, 2010, 2(2): 23-26.

[8]Xu Guobao, Yin Yixin. Visual information processing using cellular neural networks for mobile robot[J]. Proceedings of 2007 IEEE International Conference on Grey Systems and Intelligent Services, Nanjing, China. 2007(11): 1046-1050

[9] RUYI J, REINHARD K, TOBI V, et al. Lane detection and tracking using a new lane model and distance transform[J]. Machine Vision and Application, 2011, 22(4): 721-737.

[10] Heechul Jung, Junggon Min and Junmo Kim. An Efficient Lane Detection Algorithm for Lane Departure Detection. 2013 IEEE Intelligent Vehicles Symposium (IV). 2013, 23(26): 976-980.

[11] Mochizuki Yoshihiko, Torii Akihiko, Imiya Atsushi. N-Point Hough transform for line detection [J]. Journal of Visual Communication and Image Representation, 2009, 20(4): 242-253.

[12] DU S, TU C, SUN M. High accuracy Hough transform based on butterfly symmetry[J]. Electronics Letters, 2012, 48(4): 199-201.

[13] GUERREIRO R F C, AGUIAR P M Q. Connectivity-enforcing Hough transform for robust extraction of line segments[J]. IEEE Transcation on Image Processing, 2012,21(12):4819-4829.

[14] HUNJAE Y, UKIL Y, KWANGHOON S. Gradient-enhancing conversion for illumination-robust lane detection[J]. IEEE Transactions on Intelligence Transportation Systems, 2013, 14(3): 1083-1094.

[15]Liu Hua-jun, Zhang Hao-feng. Quantitative evaluation and information fusion of road edges for accurate unstructured road tracking $[\mathrm{J}]$. 2006 6th International Conference on ITS Telecommunications Proceedings. 2006: 318-321 